\title{
The Influence of Background Objects on Unpleasantness Evoked by Lotus-seed-pods-on-the-living-body Images ("Hasu-colla")
}

\author{
Manami FURUNO*, Shu IMAIZUMI**, Kana MAEDA*, \\ Haruo HIBINO* and Shinichi KOYAMA* \\ * Chiba University, 1-33 Yayoi-cho, Inage-ku, Chiba 263-8522, Japan \\ ** University of Tokyo, 3-8-1 Komaba, Meguro-ku, Tokyo 153-8902, Japan
}

\begin{abstract}
The photomontage of lotus seedpods on human skin has been called "Hasu-colla" in Japan, and this can elicit strong aversion in viewers. Previous studies have reported that lotus seedpods evoke core disgust, and that Hasu-colla relates to animal reminder disgust. However, the relationship between unpleasantness evoked by lotus seedpods and that by Hasu-colla has not been demonstrated. The present study investigated whether Hasu-colla evokes stronger disgust than lotus seedpods presented alone as well as differences between background objects (from animal to stone) in evoking aversion. Hasu-colla induced stronger unpleasantness than did lotus seedpods and background objects presented alone (Exp. 1 and 2). The amplification of unpleasantness was weakest in stones and strongest in dogs among animals (Exp. 2). Lotus seedpods, which are related to disgust for clusters, evoke strong feelings of contamination, supporting the possibility that such clusters are associated with scars and sores.
\end{abstract}

Keywords: Hasu-colla (Lotus-seed-pods-on-the-living-body), Disgust, Trypophobia

\section{INTRODUCTION}

The photomontage of lotus seedpods (the part with no petal, stamen, or pistil) on skin has been called "Hasu-colla" in Japan (Hasu means lotus in Japanese while Colla is an abbreviation of collage). It is reported that this evokes strong aversion for many observers [1]. Disgust evoked by Hasu-colla can be explained by two factors.

First, we propose trypophobia. This refers to disgust regarding a circular cluster such as lotus seedpods [2, 3], and these are typical stimuli that induce trypophobia [4]. A recent study suggests that trypophobia is evoked by not only circular clusters but also clusters of eyes and faces [5]. Trypophobia is predicted by core disgust [6], which is one factor of disgust sensitivity [7]. This refers to a tendency to experience disgust. It acts as a defense against contamination and disease [8]. In a previous study, it was shown that Hasu-colla is related to disgust sensitivity (especially animals and death). It has been proposed that lotus seeds are associated with maggots on a dead body [9]. Thus, we hypothesized that when unpleasant lotus seedpods are shown on specific background objects (e.g., human; we defined the combination of lotus seedpods and background objects as Hasu-colla, Figure 1), stronger unpleasantness will be evoked than when only lotus seedpods are viewed, and that this phenomenon is Hasu-colla.
Second, studies have also shown that empathy for pain partially contributes to the disgust evoked by Hasu-colla. For example, researchers have suggested that lotus seedpods might be associated with scars and sores [3]. When unpleasant lotus seedpods are placed on a living object (e.g., human), we predict that observers will tend to imagine others' disease and pain. Recent studies have indicated that empathy for pain contributes to an estimation of others' pain [10-12]. Moreover, brain areas implicated in pain and that mediate empathy for pain are more active when observing in-group members as compared to out-group members [13]. Thus, we hypothesized that observers are more empathetic towards imagined pain of the same species' Hasu-colla. In other words, lotus seedpods on human skin (human Hasu-colla) may elicit a stronger aversion than lotus seedpods on animal skin (animal Hasu-colla).

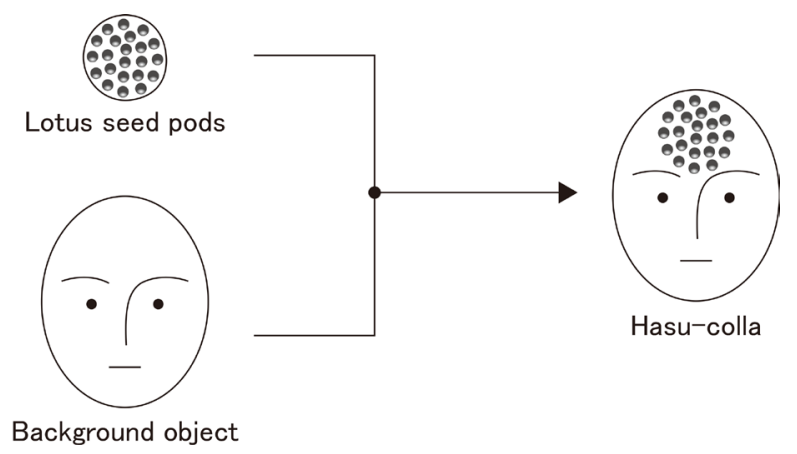

Figure 1: We defined Hasu-colla as placing lotus seedpods on a background object. 
The present study aimed to test the above two hypotheses. First, we examined whether people feel stronger disgust when lotus seedpods are presented in combination with a background object than when lotus seedpods are presented alone. Second, we examined whether unpleasantness is more amplified when lotus seedpods are placed on a human rather than an animal as the background object. In Experiment 1, using various human and animal Hasu-colla and corresponding images of only lotus seedpods, we compared the unpleasantness between images of only lotus seedpods and each Hasu-colla, and then compared the quantitative amplification of unpleasantness between human and animal Hasu-colla. We speculated that images with lotus seedpods induce stronger unpleasantness than images of only lotus seedpods and the amplification quantity of unpleasantness is higher in humans than animals. In Experiment 2, we selected images of humans, dogs, lions, plants, and stones in order of likelihood to empathize for pain as well as compared unpleasantness when lotus seedpods were placed, to review Experiment 1 in detail. We considered whether the amplified unpleasantness evoked by placing lotus seedpods is specific to humans or also occurs in animals, plants, and stones. If the unpleasantness evoked by placing lotus seedpods occurs in animals as well as humans, we assumed that the face inversion effect, which decreases face recognition, would reduce this unpleasantness.

There are many people who feel unpleasant while viewing Hasu-colla [1] However, to our knowledge, no empirical study has shown whether images depicting lotus seedpods placed on a background object increase unpleasantness of viewing and what factors are influential in the amplification of unpleasantness in the Hasu-colla. By elucidating the mechanism underlying unpleasantness evoked by placing lotus seedpods on an object, we may help to develop interventions for unpleasantness evoked by Hasu-colla and/or clusters of objects seen in daily life (e.g., polka-dots, lotus seedpods).

\section{EXPERIMENT 1}

We conducted pleasantness-unpleasantness ratings for human and animal Hasu-colla to examine the amplification of unpleasantness by placing lotus seedpods on background objects, and whether the quantity of amplification of unpleasantness was highest in human Hasu-colla.

\subsection{Methods}

\subsubsection{Participants}

Thirteen adults (nine females; mean age $21.9 \pm 0.76$ years) participated in the experiment. Written informed consent was obtained from each participant. The experiments were conducted according to the principles of the Declaration of Helsinki and approved by the ethical committee of the Graduate School of Engineering, Chiba University.

\subsubsection{Apparatus and stimuli}

The stimuli consisted of forty images. Twenty images were Hasu-colla, ten each of either humans or animals. The former consisted of men and women who were young adults or elderly, and half of the latter consisted of large animals (e.g., horse) and the other half of small animals (e.g., cat). All of these images contained faces and upperlimbs on backgrounds of natural or urban scenes. Lotus seedpods were placed in various locations such as face or upper-limbs of the human and animal images in order to reconstruct the known Hasu-colla that typically display lotus seedpods on an arbitrary body part, such as the face and forearm. Images of only lotus seedpods corresponded to each of the ten human and animal images (these images were selected from Google Images). Figure 2 is a sample of the stimuli. Size and collage positions of the lotus seedpods were adjusted to a suitable size and position to fit into parts of the face or limbs for each background object. Each stimulus was full-color and the average luminance was $6.65 \mathrm{~cd} / \mathrm{m}^{2}(\mathrm{SD}=0.57)$. The images of only lotus seedpods were placed against a gray background using Adobe Photoshop CS6. The luminance of the gray background matched the luminance of each background

(a)

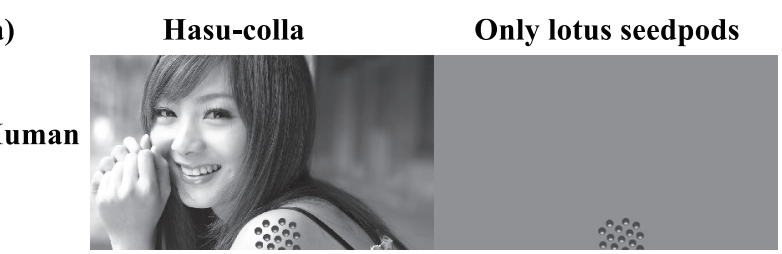

(b)

Animal

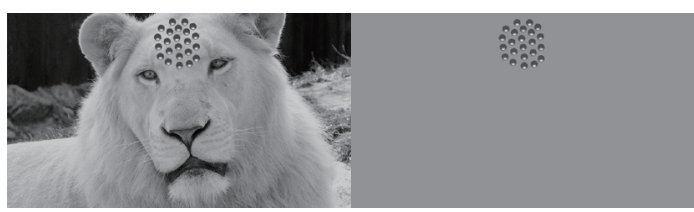

Figure 2: Samples of stimuli used in Exp 1. (a) Left (Original image; 201005132000 by Patrick available at http://free.gatag.net/2010/05/13/200000.html under CC BY-SA 4.0.) is human Hasu-colla; the Right shows a reproduction of an image of only lotus seedpods. (b) Left (Original image; ZOO Bratislava - African Lion (Panthera leo krugeri) by Stano Novak available at https://commons.wikimedia.org/wiki/File:White_Lion.jpg under CC BY-SA 2.5.) is animal Hasu-colla; Right is a reproduction of only the lotus seedpods. Please note that the samples of the images of only lotus seedpods are $\mathrm{CG}$ reproductions. We used realistically depicted photos for images of only lotus seedpods in the actual experiment. 
object. Stimuli were presented $20.8^{\circ} \times 36.1^{\circ}$ in a darkened room. Participants rested on a chin rest and viewed the stimuli. We used a 24-inch monitor (ASUS VG248QE with a resolution of $1920 \times 1080 \mathrm{px})$ and programmed with Hot Soup Processer (HSP) 3.4 for displaying stimuli.

We conducted a pilot study and controlled unpleasantness between humans and animals without the lotus seedpods (original images) as well as between images of only lotus seedpods used for human and animal images to determine whether unpleasantness is influenced by differences in background objects. Eight (4 female; mean age $22.0 \pm 1.50$ years) and six (4 female; mean age $23.0 \pm 1.50$ years) adults participated in the pilot studies for original images (human and animals) and images only of lotus seedpods used for humans and animals, respectively. Pilot study participants did not participate in the main experiment (Experiment 1). Two-tailed t-tests revealed no significant difference between the original images of humans and animals $(t(7)=0.76, p=.47$, $d=0.32$ ), or between images only of lotus seedpods used for humans and animals $(t(5)=0.24, p=.83, d=0.08)$.

\subsubsection{Procedure}

The experiment consisted of a practice task and a rating task. Participants were told that there was a possibility that they would find images in the experiment unpleasant (a set of images of only lotus seedpods and each Hasucolla), and that they could stop at any time. Those who agreed to these instructions participated. After a fixation point was presented in the center of the monitor for $750 \mathrm{~ms}$, the instruction "Please push a number to indicate how you felt when you viewed the above image" was displayed with stimuli that were unrelated to the rating task. The observers were asked to rate using a 7-point scale that ranged from " -3 : extremely repulsive," "-2: repulsive," "-1: unpleasant," "0: neither unpleasant nor pleasant," "1: pleasant," "2: attractive," to "3: extremely attractive" using the numeric keypad. This trial was performed twice.

The rating task was completed after the practice task. After the fixation point was presented for $750 \mathrm{~ms}$, one of the forty images was displayed in a random order. These images included ten each of human Hasu-colla, animal Hasu-colla, lotus seedpods used in the human Hasu-colla, and lotus seedpods used in the animal Hasu-colla. Images of lotus seedpods were on a gray uniform background. Identical to the practice task, the instruction "Please push a number to indicate how you felt when you viewed the above image" was presented, and participants were asked to rate using a 7-point scale identical to the one used in the practice task. There was an inter-trial interval of $500 \mathrm{~ms}$ for each trial during the rating task.

\subsection{Results}

The results are shown in Figure 3. Two-tailed t-tests for average pleasantness scores of human Hasu-colla, animal Hasu-colla, and images of only lotus seedpods revealed significant differences between human Hasu-colla and images of only lotus seedpods $(t(12)=-5.55, p<.001$, $d=1.51$ ), and between animal Hasu-colla and images of only lotus seedpods $(t(12)=-4.36, p<.001, d=1.21)$. Figure 4 shows the mean rating differences of images of only lotus seedpods used in human/animal images and Hasu-colla of each category. One-sample two-tailed t-tests revealed that both human and animal images significantly differed from zero (human: $t(12)=-5.55, p<.001$, $d=2.08$; animal: $t(12)=-4.36, p<.001, d=1.73$ ), and there was no significant difference between humans and animals $(t(12)=-0.32, p=0.75, d=0.10)$.

\subsection{Discussion}

The results suggested that unpleasantness is amplified when images of lotus seedpods used for humans and animals are shown, compared with lotus seedpods alone. This is consistent with our hypothesis that unpleasantness is amplified when lotus seedpods images are placed on background objects.

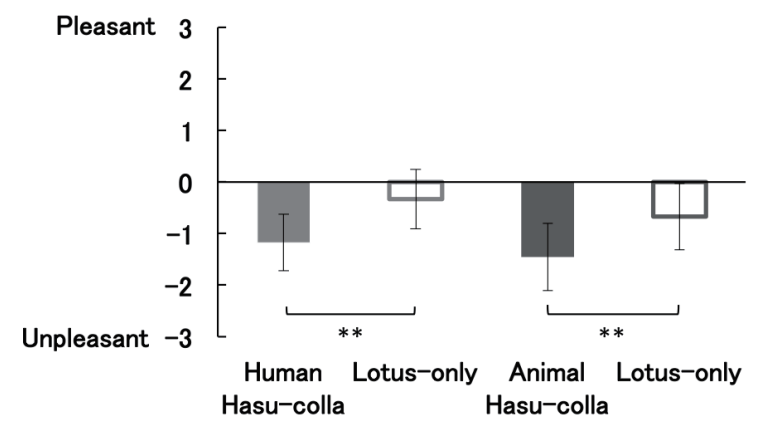

Figure 3: The mean ratings of human Hasu-colla/animal Hasu-colla/ only lotus images corresponding with each other. Error bars denote standard errors of the mean. ${ }^{*} p<<.01$

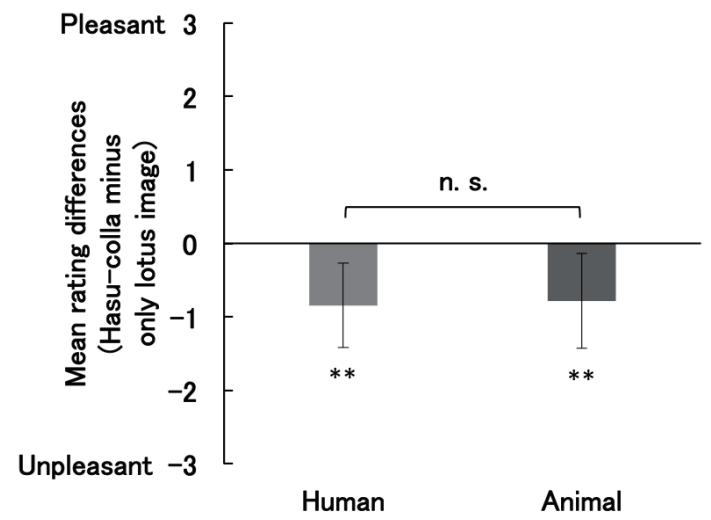

Figure 4: The values of mean rating differences between only lotus seedpod and human/animal images. Error bars denote standard errors of the mean. $* * p<.01$ 
A previous study suggested that clusters such as lotus seedpods relate to core disgust [6]. Core disgust is elicited by the threat of contamination (e.g., rotting food and body secretions) [14]. When lotus seedpods, which are associated with contamination, are placed on a background object, the feeling of contamination might amplify feelings of unpleasantness. Moreover, disgust sensitivity relates to not only core disgust but also animal reminder disgust. Animal reminder disgust is induced by damaged bodies and death [14]. A previous study suggested that Hasu-colla might be associated with maggots on a dead body [9]. Thus, Hasu-colla elicits more aversion than lotus seedpods alone because animal reminder disgust and core disgust are evoked simultaneously by placing lotus seedpods on an animal.

Experiment 1 did not indicate a significant difference in the amplification of unpleasantness between human and animal backgrounds with a photomontage of lotus seedpods. It is possible that we could not examine differences between humans and animals in detail due to differences in the form, size, density, and placement position of lotus seedpods in each image.

Next, we considered whether the background object being an animal in addition to being human specifically evokes strong aversion. If lotus seedpods are closely related to animal reminder disgust, unpleasantness may differ between animals and non-animals. This is suggested by the idea that when lotus seedpods are placed on an animal (animal Hasu-colla), they are easily associated with a damaged body, i.e., disease and scars, thereby inducing stronger feelings of unpleasantness compared to non-animal images. To investigate this hypothesis, we conducted Experiment 2 using a wide range of stimuli.

\section{EXPERIMENT 2}

To consider differences in unpleasantness for background objects in detail, we used one of the images of the lotus seedpods and regulated its size and position. We also selected images of humans, dogs, and lions as animals in order of likelihood to empathize for pain. We anticipated that human images would be likely to evoke the most empathy for pain and unpleasantness. In contrast, lion images are likely to evoke the least empathy for pain and unpleasantness. Furthermore, images of plants that can be eaten such as vegetables and fruits, and stones were added as non-animals. We assumed that Hasu-colla of these images would evoke less unpleasantness than animal images such as those of humans, dogs, and lions because these non-animal images are likely to evoke less animal reminder disgust. Furthermore, we also anticipated that plant Hasu-colla might increase unpleasantness particularly when the plant images depict edible plants, because disgust involves food rejection as its core function and could be enhanced by rotten and/or contaminated food [15]. It was expected that non-animal images, especially those displaying stones would evoke less unpleasantness than animal images, while non-animal images of edible plants would increase unpleasantness when lotus seedpods are placed on them, which may be associated with rot and/or contamination. Therefore, we included images of edible plants as stimuli in this experiment.

We also predict that the special perception of human faces [16] might act to amplify unpleasantness, as we placed lotus seedpods on the forehead, which is a part of the face. It is possible that the amplification of unpleasantness by lotus seedpods is lower when face recognition is disturbed. It has been widely reported that face recognition is impaired when the face is inverted [17]. For example, inverted disgust faces elicit smaller N170 event-related potentials than upright disgust faces [18]. The early posterior negativity (EPN) shows similar results. The EPN relates to emotional experience and selective attention $[19,20]$ and responds stronger to disgust-related stimuli such as snakes [21,22]. This suggests that disgust for lotus seedpods on faces might be reduced with stimuli inversion. Thus, we examined the relationship between unpleasantness (by placing lotus seedpods on faces) and face recognition. It is known that the inversion effect can occur in a face but less in other objects [23]. This is also reported repeatedly in other experiments [24,25]. Hence, we inverted only humans, dogs, and lions, i.e., stimuli that have a face. These inverted versions of humans/dogs/lions were added to upright versions of images of humans/dogs/lions/ plants/ stones, and we compared the unpleasantness of these images.

\subsection{Methods}

\subsubsection{Participants}

Nineteen adults (twelve females; mean age 19.3 \pm 0.48 years) who were not recruited for Experiment 1 participated in the experiment.

\subsubsection{Apparatus and stimuli}

We used ninety-six images as stimuli. The upright original images of humans/dogs/lions/plants/stones and their corresponding Hasu-colla, for which we used the same lotus seedpods, as well as the inverted original images of human/dogs/lions and their corresponding 
Hasu-colla, each comprised six images. Each human image consisted of a male face. The dog images were heads of large dogs. The lion images were a mix of male and female heads. Each plant and stone image displayed a circular shape so that it would be similar to the shape of a face. The plants consisted of vegetables and fruits. The plants and stones were distinguished by "whether it can metabolize on its own." The images of humans, dogs, and lions contained a face as subjects and the images of lotus seedpods were placed on the forehead of each animal subject. In the plants and stones, lotus seedpods were placed on the upper part of each object in order to resemble humans, dogs, and lions. Figure 5 is a sample of the stimuli. The average luminance was $21.62 \mathrm{~cd} / \mathrm{m}^{2}(\mathrm{SD}=0.37)$. These were presented in the same size and apparatus as Experiment 1. We conducted a pilot study and controlled the unpleasantness of each upright original image.
Seven adults (three females, mean age $22.4 \pm 0.79$ years) who did not participate in Experiment 1 or the main experiment (Experiment 2) performed the pleasantnessunpleasantness evaluation in the pilot study. No difference in the upright images of humans/dogs/ lions/plants/stones were found by a series of one-way ANOVAs performed with image category as a factor $(F(4,25)=2.10, p=.11$, $\left.\eta^{2}=.25\right)$. Moreover, one-sample two-tailed t-tests revealed that the upright images did not significantly differ from zero.

\subsubsection{Procedure}

This experiment was identical to Experiment 1 except for the stimuli presented in the rating task. Six each of the upright images of humans/dogs/lions/plants/stones and inverted images of humans/dogs/lions, and the corresponding Hasu-colla totaled ninety-six images, which were displayed in a random order. (a)

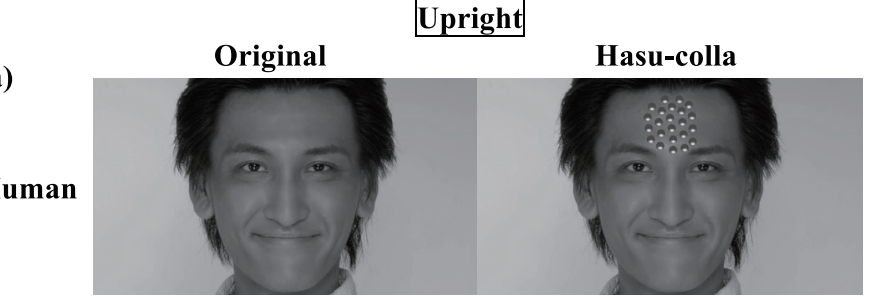

(b)

Dog

(c)

Lion

(d)

Plant

(e)
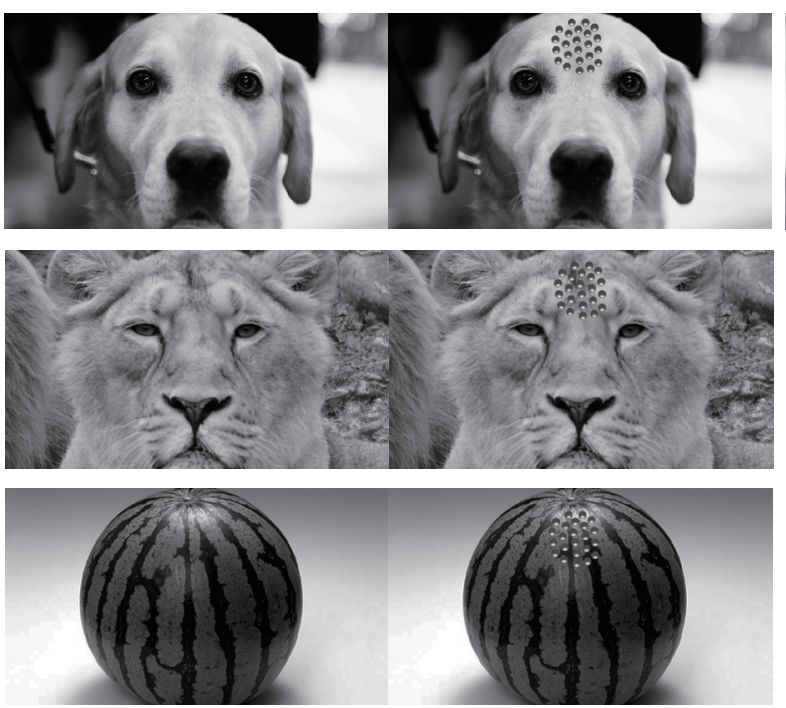

Stone

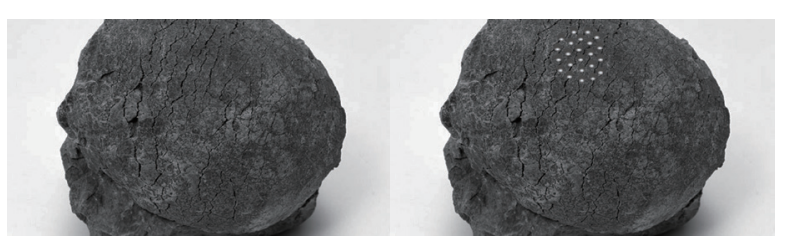

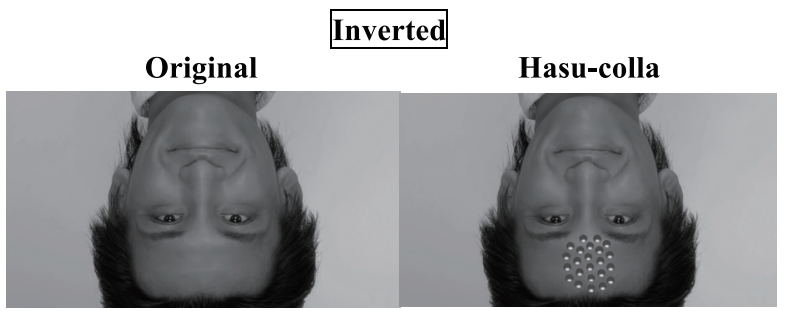


Figure 5: Samples of stimuli used in Exp 2. (a) Left is original human; Right is a reproduction of human Hasu-colla. (b) Left is original dog; Right is a reproduction of dog Hasu-colla. (c) Left is original lion; Right is a reproduction of lion Hasu-colla. (d) Left is original plant; Right is a reproduction of plant Hasu-colla. (e) Left is original stone; Right is a reproduction of stone Hasu-colla. There are two types of upright and inverted images for humans/dogs/plants. Please note that the parts with lotus seedpods are reproduction by CG. We used realistically depicted photos as images of only lotus seedpods in the actual experiment. 


\subsection{Results}

Figure 6 presents results that indicate the mean pleasantness ratings of upright and inverted Hasu-colla of humans/dogs/lions/plants/stones and the original images of each category. Two-tailed t-tests revealed a significant difference between upright Hasu-colla of all categories and their corresponding original images (humans: $t(18)=-6.93, p<.01, d=2.07$; dogs: $t(18)=-7.18$, $p<.01, d=2.44$; lions: $t(18)=-6.30, p<.01, d=1.96$; plants: $t(18)=-4.97, p<.01, d=1.69$; stones: $t(18)=-4.50$, $p<.01, d=1.05)$. Moreover, Figure 7 shows the mean differences in pleasantness ratings of upright and inverted original images of humans/dogs/lions/plants/stones and the Hasu-colla of each category. A series of one-way ANOVAs performed on scores with upright image category as a factor revealed a significant main effect $\left(F(4,25)=7.31, p<.001, \eta^{2}=.56\right)$. Multiple comparisons using Tukey's b indicated that dogs-humans, dogs-lions, dogs-stones, and plants-stones were significant.

Two-tailed t-tests also revealed a significant difference between all inverted Hasu-colla of humans/dogs/lions and the inverted original images of each category (humans: $t(18)=4.91, p<.001, d=1.57$; dogs: $t(18)=5.24$, $p<.001, d=1.55$; lions: $t(18)=5.80, p<.001, d=1.71)$. A series of two-way ANOVAs was performed on the rating differences for upright/inverted Hasu-colla and corresponding upright/inverted original images, with image category and upright vs. inversion as factors. The results showed no significant interaction between category and upright vs. inversion $\left(F(2,30)=1.68, p=.20, \eta_{p}{ }^{2}=.10\right)$. However, there was a significant main effect for upright vs. inversion $\left(F(1,30)=12.6, p<.001, \eta_{p}{ }^{2}=.30\right)$. The results indicated no significant main effect for image category $\left(F(2,30)=2.22, p=.13, \eta_{p}{ }^{2}=.13\right)$.

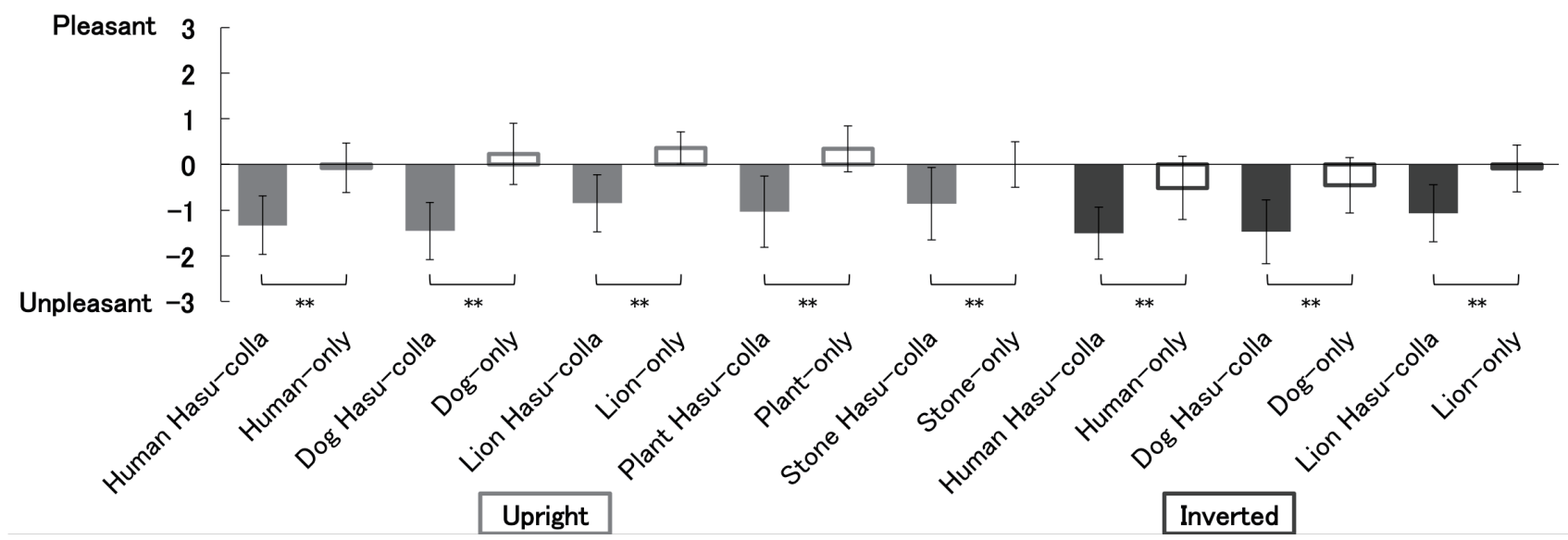

Figure 6: The mean ratings of upright and inverted Hasu-colla and original humans/dog/lion/plant/stone images. Error bars denote standard errors of the mean. ${ }^{* *} p<.01$

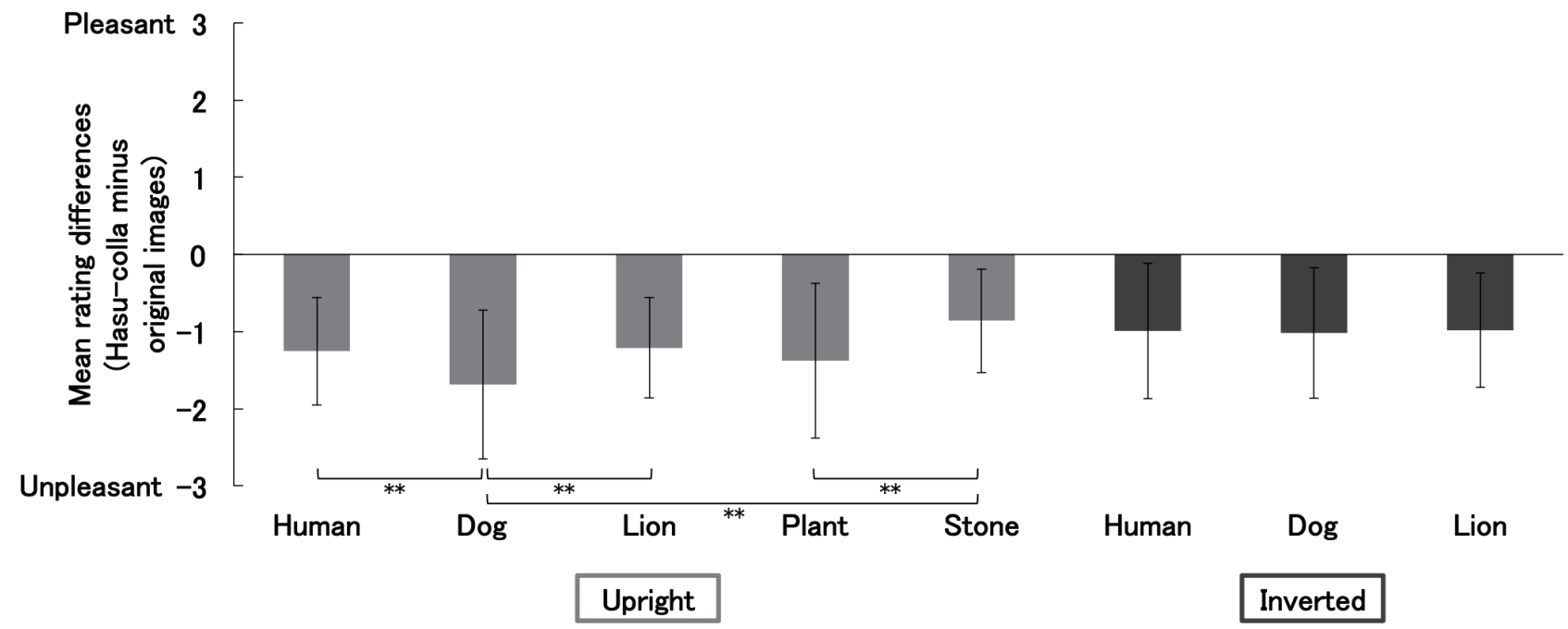

Figure 7: The mean rating differences for upright and inverted Hasu-colla and original humans/dogs/lions/plants/stone images. Error bars denote standard errors of the mean. ${ }^{* *} p<.01$ 


\subsection{Discussion}

Results suggested that unpleasantness was evoked by adding lotus seedpods regardless of whether the image was an animal or not. If lotus seedpods induce core disgust, it is expected that both animals and non-animals will become contaminated, thereby contributing to all background objects becoming aversive. Contamination renders liked foods inedible [15]. Lotus seedpods are a disgust elicitor that induce contamination.

Placing lotus seedpods on stones showed the least amplification of unpleasantness. If lotus seedpods are placed on animals such as humans/dogs/lions, this elicits animal reminder disgust, i.e., damaged bodies and death. In contrast, if lotus seedpods are placed on non-animals, it might elicit less animal reminder disgust, and these results support our hypothesis. However, plants did not support the above hypothesis, as the unpleasantness for plant Hasu-colla was the strongest after dog Hasu-colla. We speculate that placing lotus seedpods on an animal may elicit an unpleasantness perhaps driven by animal reminder disgust when placing it on non-animals, but edible objects may elicit unpleasantness driven by core disgust originating from a rejection of contaminated food. The Disgust and Contamination Sensitivity Questionnaire (DQ; the first questionnaire on disgust sensitivity) [26] measures disgust sensitivity to contaminated foods, and it has been suggested that disgust has origins in food rejection [15]. Lotus seedpods, which evoke core disgust, might induce primitive disgust and strong aversion when placed on plants consisting of edible foods such as fruits and vegetables.

Moreover, the amplification of unpleasantness by placing lotus seedpods on the face was demonstrated in inverted images. However, the amplification of unpleasantness was less in inverted than in upright face images. Nevertheless, the contamination effect of lotus seedpods was demonstrated even in inverted face images with decreased recognition [17], and it is possible that aversion decreased with a decrease in facial recognition.

Dog Hasu-colla evoked more unpleasantness than human and lion Hasu-colla in Experiment 2. It was thought that the ease with which pain can be empathized would be heightened in same-species judgments, making it difficult to explain the unpleasantness ratings for dogs. Moreover, in terms of disgust sensitivity such as core disgust and animal reminder disgust, it is also difficult to explain this result because humans, dogs, and lions are all animals. Thus, it is possible that other factors contribute to the unique unpleasantness of dog Hasu-colla. Further studies should examine factors beyond disgust sensitivity that might be affected by Hasu-colla.

\section{GENERAL DISCUSSION}

The results suggest that Hasu-colla induce more aversion than lotus seedpods or background objects alone (Exp.1 and 2), and dogs as background objects lead to greater feelings of unpleasantness as compared to humans as background objects (Exp. 2). Aversion caused by lotus seedpods on the faces of animals was less when the faces were inverted than when upright. Moreover, the unpleasantness elicited by lotus seedpods on food was comparable with that on animals.

First, Hasu-colla evoked unpleasantness regardless of background object type. The effect can be explained by core disgust. Core disgust is a part of disgust emotion elicited by something involving contamination [14]. Imaizumi et al. (2016) showed that trypophobia is predicted partially by core disgust [6]. Moreover, lotus seedpods are typical stimuli that induce trypophobia [4]. The visual appearance of lotus seedpods looks similar to scars and sores, and therefore, may remind viewers of them [3]. Thus, it is possible that placing lotus seedpods on background objects leads to feelings of contamination and induces disgust. Such a spread of contamination appears to follow the law of contamination of Magic [27]: "once in contact, always in contact."

Second, differences in the amplification of unpleasantness among background objects can be explained by animal reminder disgust and disgust to food contamination. Animal reminder disgust is induced by damaged bodies and death [14]. If clusters such as lotus seedpods are associated with scars and sores [3], this could involve imagining scars and sores in lotus seedpods on the skin of animals. This may induce animal reminder disgust, a suggestion in accordance with past studies. Skaggs (2014) suggested that when people prone to trypophobia view cluster images, especially images of holes in the skin, they produce skin crawling [3]. Blake et al. (2016) indicated that aversive clusters that resemble skin lesions tend to elicit heebie-jeebies related to skin crawling and goosebumps [28]. Wada (2012) reported that aversion to Hasu-colla reminds of disgust for animals and dead bodies, and assumed that Hasu-colla is associated with maggots on a dead body [9]. Furthermore, Wada (2016) conducted an experiment similar to the present study [29]. The results coincide with the present study, indicating that placing lotus seedpods on the back of the hand induces stronger aversion as compared to lotus seedpods alone or placing lotus seedpods on bark. These studies verify our hypothesis that animal reminder 
disgust, such as disgust with damaged bodies and death, is elicited by placing lotus seedpods on animals, and leads to strong feelings of unpleasantness as compared to placing lotus seedpods on stones. The inversion effect seen in humans and animals also supports this hypothesis. Unpleasantness was reduced when faces were inverted. If inversion reduces face recognition, the humanity and animality of the faces may have decreased as well. Furthermore, plant stimuli, which consisted of food such as vegetables and fruits, induced comparable aversion to animal Hasu-colla. If lotus seedpods are contamination elicitors, placing lotus seedpods on food may lead people to think the food is contaminated. We speculate that core disgust originating from the recall of food contamination [15] might be a source of the strong disgust. Indeed, experimental evidence has suggested that, for example, individuals tend to reject a liked beverage after it comes into contact with an unsavory object (e.g., cockroach) $[15,30]$. Nevertheless, further research is needed to investigate whether the Hasu-colla for edible plants entail a contamination-based disgust.

There are two limitations of this study. First, we cannot explain unpleasantness differences between dog Hasu-colla and human/lion Hasu-colla by animal reminder disgust because these are all categorized as animals. We should investigate other factors in explaining this issue. Second, it is possible that the differences in unpleasantness could be explained by the spatial frequency of background objects. Cole and Wilkins (2013) and Le et al. (2015) indicated that trypophobic images have characteristic spatial frequency properties $[2,4]$. We partly regulated the luminance, but not spatial frequency, in Experiments 1 and 2. Thus, a study focusing on the relationship between spatial frequency and unpleasantness as a function of lotus seedpods should be conducted.

To conclude, our results suggested that the disgust for a cluster of holes (i.e., trypophobia) can be enhanced when the cluster (e.g., lotus seedpods) is placed on animals or non-animal objects. Importantly, there might be different underlying mechanisms; lotus seedpods on animals elicit animal reminder disgust, while lotus seedpods on an edible plant (i.e., a non-animal object) elicit core disgust. Given that the substantial inter-individual variability of Hasu-colla-evoked disgust [9] and trypophobia [4, 6, 31], there may be a demand for an effective intervention for those susceptible to these disgust responses. Our results that suggest that the negative cognitive processing associated with scars, sores and food rejection might contribute to the unpleasantness of placing lotus seed- pods on background objects (i.e., Hasu-colla), and also imply that people who are sensitive to Hasu-colla and/or clusters need help. This is because attributing excessive significance such as associations of clusters to skin scars and sores is often seen in other phobia patients [32, 33]. Thus, it is possible that people who feel strong unpleasantness to Hasu-colla and/or clusters require support to relieve unpleasantness like other phobia patients. For example, design guidelines to reduce visual disgust elicitors, particularly in public spaces. We hope that our findings and future studies could help to respond such demand and to understand Hasu-colla-evoked disgust and trypophobia more deeply in terms of psychology and affective engineering.

\section{ACKNOWLEDGEMENTS}

Experiment 1 was part of an unpublished graduation thesis by KM. This work was supported by a Grant-in-Aid for JSPS Fellows from the Japan Society for the Promotion of Science to SI (13J00943).

\section{REFERENCES}

1. Y. Yamada; Kimochiwarusa no Shinrigaku, In; K. Miura (Ed.), Kanse-ninchi: Aisthesis no Shinrigaku, Kitaoji Shobo, Kyoto, pp.151, 2016. (In Japanese)

2. G.G. Cole, A.J. Wilkins; Fear of holes, Psychological Science, 24(10), pp.1980-1985, 2013.

3. W. Skaggs; Fear of holes, Scientific American Mind, 25(2), p.12, 2014.

4. A.T. Le, G.G. Cole, A.J. Wilkins; Assessment of trypophobia and an analysis of its visual precipitation, Quarterly Journal of Experimental Psychology, 68(11), pp.2304-2322, 2015.

5. K. Chaya, Y. Xue, Y. Uto, Q. Yao, Y. Yamada; Fear of eyes: triadic relation among social anxiety, trypophobia, and discomfort for eye cluster, PeerJ, 4, p.e1942, 2016.

6. S. Imaizumi, M. Furuno, H. Hibino, S. Koyama; Trypophobia is predicted by disgust sensitivity, empathic traits, and visual discomfort, SpringerPlus, 5(1), pp.1-5, 2016.

7. J. Haidt, C.R. McCauley, P. Rozin; Individual differences in sensitivity to disgust: A scale sampling seven domains of disgust elicitors, Personality and Individual Differences, 16(5), pp.701-713, 1994.

8. S.R. Woody, B.A. Teachman; Intersection of Disgust and Fear: Normative and Pathological Views, Clinical Psychology: Science and Practice, 7(3), pp.291-311, 2006. 
9. Y. Wada; Disgust sensitivity in "Hasu-colla" (photomontage of human body and lotus seeds) and other disgust elicitors, 76th Annual Convention of the Japanese Psychological Association, Kawasaki, 2012.

10. A.D. Green, D.A. Tripp, M.J. Sullivan, M. Davidson; The relationship between empathy and estimates of observed pain, Pain Medicine, 10(2), pp.381-392, 2009.

11. N. Danziger, K.M. Prkachin, J.C. Willer; Is pain the price of empathy? The perception of others' pain in patients with congenital insensitivity to pain, Brain, 129(Pt 9), pp.2494-2507, 2006.

12. M.J. Giummarra, B.M. Fitzgibbon, N. GeorgiouKaristianis, M. Beukelman, A. Verdejo-Garcia, Z. Blumberg, M. Chou, S.J. Gibson; Affective, sensory and empathic sharing of another's pain: The Empathy for Pain Scale, European Journal of Pain (London, England), 19(6), pp.807-816, 2015.

13. X. Xu, X. Zuo, X. Wang, S. Han; Do you feel my pain? Racial group membership modulates empathic neural responses, Journal of Neuroscience, 29(26), pp.8525-8529, 2009.

14. P. Rozin, J. Haidt, C.R. McCauley; Disgust; In; M. Lewis, J.M. Haviland-Jones and L.F. Barrett (Ed.) Handbook of emotions, Guilford Press, New York, 2008.

15. P. Rozin, A.E. Fallon; A perspective on disgust, Psychological Review, 94(1), pp.23-41, 1987.

16. N. Kanwisher, J. McDermott, M.M. Chun; The fusiform face area: a module in human extrastriate cortex specialized for face perception, Journal of Neuroscience, 17(11), pp.4302-4311, 1997.

17. T. Valentine; Upside-down faces: A review of the effect of inversion upon face, British Journal of Psychology, 79(4), p.471, 1988.

18. V. Ashley, P. Vuilleumier, D. Swick; Time course and specificity of event-related potentials to emotional expressions, Neuroreport, 15(1), pp.211-216, 2004.

19. H.T. Schupp, J. Markus, A.I. Weike, A.O. Hamm; Emotional Facilitation of Sensory Processing in the Visual Cortex, Psychological Science, 14(1), pp.7-13, 2003.

20. H.T. Schupp, T. Flaisch, J. Stockburger, M. Junghöfer; Emotion and attention: event-related brain potential studies, G.E.M.J.J.K. S. Anders and D. Wildgruber (Ed.) Progress in Brain Research, Elsevier, pp.31-51, 2006.

21. J.W. Van Strien, R. Eijlers, I.H.A. Franken, J. Huijding; Snake pictures draw more early attention than spider pictures in non-phobic women: Evidence from eventrelated brain potentials, Biological Psychology, 96, pp.150-157, 2014.

22. J.W. Van Strien, I.H.A. Franken, J. Huijding; Testing the snake-detection hypothesis: Larger early posterior negativity in humans to pictures of snakes than to pictures of other reptiles, spiders and slugs, Frontiers in Human Neuroscience, 8, 2014.

23. R.K. Yin; Looking at upside-down faces, Journal of Experimental Psychology, 81(1), p.141, 1969.

24. T. Valentine, V. Bruce; The effects of distinctiveness in recognising and classifying faces, Perception, 15(5), pp.525-535, 1986.

25. R. Diamond, S. Carey; Why faces are and are not special: an effect of expertise, Journal of Experimental Psychology: General, 115(2), p.107, 1986.

26. P. Rozin, A. Fallon, R. Mandell; Family resemblance in attitudes to foods, Developmental Psychology, 20(2), pp.309-314, 1984.

27. J.G. Frazer; The new golden bough: A study in magic and religion (abridged), Macmillan, New York, 1959.

28. K.R. Blake, J. Yih, K. Zhao, B. Sung, C. Harmon-Jones; Skin-transmitted pathogens and the heebie jeebies: evidence for a subclass of disgust stimuli that evoke a qualitatively unique emotional response, Cognition and Emotion, pp.1-16, 2016.

29. Y. Wada; Does disgust sensitivity to HASU-COLLA (photomontage of lotus seeds on human skin) correlate to general disgust sensitivity and individual differences in empathy?, 31st International Congress of Psychology, Yokohama, 2016.

30. P. Rozin, L. Millman, C. Nemeroff; Operation of the Laws of Sympathic Magic in Disgust and Other Domains, Journal of Personality and Social Psychology, 50(4), pp.703-712, 1986.

31. S. Imaizumi, M. Furuno, H. Hibino and S. Koyama; Development of the Japanese Version of Trypophobia Questionnaire, The Japanese Journal of Personality, 25(2), pp.171-173, 2016.

32. J.H. Riskind, R. Moore, L. Bowley; The looming of spiders: The fearful perceptual distortion of movement and menace, Behaviour Research and Therapy, 33(2), pp.171-178, 1995.

33. M.W. Vasey, M.R. Vilensky, J.H. Heath, C.N. Harbaugh, A.G. Buffington, R.H. Fazio; It was as big as my head, I swear!: Biased spider size estimation in spider phobia, Journal of Anxiety Disorders, 26(1), pp.20-24, 2012. 


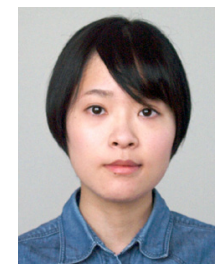

Manami FURUNO (Non-member)

She completed her B.E. from the Faculty of Engineering, Chiba University, and is presently a master course student at the Design Psychology Unit (DPU), Department of Design Science, Graduate School of Engineering, Chiba University. Her research interests are disgust, and visual cognition.

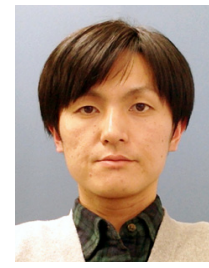

\section{Shu IMAIZUMI (Non-member)}

$\mathrm{He}$ is a JSPS Postdoctoral Research Fellow at the Graduate School of Arts and Sciences, The University of Tokyo. He received his Ph.D. from Chiba University. His research focuses on multisensory interaction and social cognition.

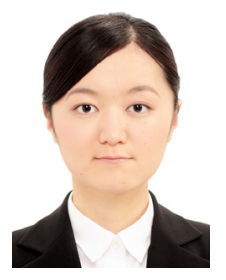

\section{Kana MAEDA (Non-member)}

She completed her B.E. from the Faculty of Engineering, Chiba University, and is currently working as a software developer.

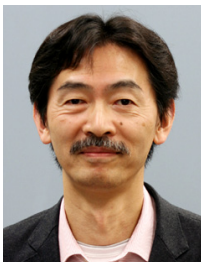

Haruo HIBINO (Member)

$\mathrm{He}$ obtained his Ph.D. degree in experimental psychology from York University, Canada, and has been working at Chiba University for nearly 27 years. He is currently a Professor in the Design Psychology Unit (DPU), Department of Design, Graduate School of Engineering, Chiba University. His research focuses on design psychology, color vision, and emotional design. He has published many papers and has contributed to many psychological books. As a chief technical advisor he is recently involved in a successful venture company (BB STONE Design Psychology Unit, Inc.) based on the intellectual property of DPU.

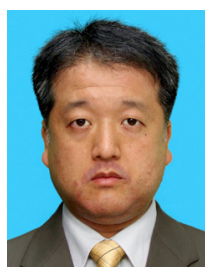

Shinichi KOYAMA (Member)

He achieved his Ph.D. from Boston University, United States. He continued his research at Boston University, Harvard Medical School, Showa University, and Nanyang Technological University. He is currently an Associate Professor in the Design Psychology Unit, Department of Design Science, Graduate School of Engineering, Chiba University, where his research focuses on cognitive psychology, neuropsychology, and design psychology. 